\title{
Erratum: Many-body diffusion and path integrals for identical particles [Phys. Rev. E 53, 4467 (1996)]
}

L. F. Lemmens, F. Brosens, and J. T. Devreese

[S1063-651X(97)02306-4]

PACS number(s): 05.30.Fk, 03.65.Ca, 02.50.Ga, 02.70.Lq, 99.10. + g

In Sec. IV D we stated that the double sum in Eq. (4.16)

$$
\int d \vec{x}^{\prime \prime} \rho_{I}\left(\bar{x}, t ; \vec{x}^{\prime \prime}, 0\right) f\left(\vec{x}^{\prime \prime}\right) \rho_{I}\left(\vec{x}^{\prime \prime}, t ; \vec{x}, 0\right)=\sum_{l, l^{\prime}=0}^{3} \int d \vec{x}^{\prime \prime} \rho_{I}\left(\bar{x}, t ; \vec{x}^{\prime \prime}, 0, l\right) f\left(\vec{x}^{\prime \prime}\right) \rho_{I}\left(\vec{x}^{\prime \prime}, t ; \vec{x}, 0 ; l^{\prime}\right)
$$

reduces to a single sum if $f\left(\vec{x}^{\prime \prime}\right)$ is invariant with respect to the permutations of the particle positions, where $\vec{x}^{\prime \prime}$ is a $3 n$-dimensional position vector of $n$ particles in a three-dimensional space. This should be corrected as follows: the double sum in Eq. (4.16) reduces to a single sum if $f\left(\vec{x}^{\prime \prime}\right)$ is invariant with respect to the permutations of the components of the particle positions. This restricts the class of scalar potentials for which the method presented in our paper can be applied without modifications, i.e., without taking into account the transitions between $\rho_{I}\left(\bar{x}, t ; \vec{x}^{\prime \prime}, 0 ; l\right)$ and $\rho_{I}\left(\vec{x}^{\prime \prime}, t ; \vec{x}, 0 ; l^{\prime}\right)$ for $l$ $\neq l^{\prime}$. Furthermore, the identity matrix $I$ should be replaced by $-I$ in the second row of the first column of the matrix in Eq. (4.8).

\section{Erratum: Viscous properties of biaxial nematic liquid crystals: The method of calculation of the Leslie viscosity coefficients [Phys. Rev. E 55, 2902 (1997)]}

\author{
[S1063-651X(97)08506-1] \\ PACS number(s): $61.30-\mathrm{v}, 66.20+\mathrm{d}, 83.70 . J \mathrm{r}, 99.10 .+\mathrm{g}$
}

Marcin Fialkowski

Equations (4.17b) and (4.17d) should read $B=-\sqrt{3}\left\langle F_{20}^{2}\right\rangle / 3+\left\langle F_{22}^{2}\right\rangle$, and $B_{1}=2 \sqrt{3}\left\langle F_{20}^{2}\right\rangle / 3$, respectively. The equations at the end of Sec. VI should have the forms

$$
1 / \tau_{1}=\frac{1}{f_{m}} \frac{3 S}{2+S}, \quad 1 / \tau_{2}=\frac{1}{f_{n}} \frac{3 B}{2+S}, \quad 1 / \tau_{3}=\frac{1}{f_{m}} \frac{3 S}{2+S} .
$$

Additionally, the expression $B_{1} \approx B$, which appears in the text just above those three equations, should be changed to $B_{1} \approx 0$. 